\title{
Effect of Yinhuangsan (YHS) on Diabetic Foot Ulcers (DFU): A Systematic Review and Meta-Analysis
}

\author{
Yichen Xiong1, Haojie Huang2, Yaochen $\mathrm{Li}^{3}$, Guohua Jiang3, Lin $\mathrm{Du}^{2 *}$ \\ ${ }^{1}$ Xinhua Hospital Affiliated to Shanghai Jiao Tong University School of Medicine, Shanghai, China \\ ${ }^{2}$ The First Affiliated Hospital of Shantou University Medical College, Shantou, China \\ ${ }^{3}$ Shenzhen Clinical Medical College, Guangzhou University of Chinese Medicine, Shenzhen, China \\ Email: ${ }^{\star 651299341 @ q q . c o m ~}$
}

How to cite this paper: Xiong, Y.C., Huang, H.J., Li, Y.C., Jiang, G.H. and Du, L. (2021) Effect of Yinhuangsan (YHS) on Diabetic Foot Ulcers (DFU): A Systematic Review and Meta-Analysis. Journal of Diabetes Mellitus, 11, 97-114.

https://doi.org/10.4236/jdm.2021.113008

Received: February 4, 2021

Accepted: August 7, 2021

Published: August 10, 2021

Copyright $\odot 2021$ by author(s) and Scientific Research Publishing Inc. This work is licensed under the Creative Commons Attribution International License (CC BY 4.0).

http://creativecommons.org/licenses/by/4.0/

\begin{abstract}
Objectives: To assess the safety and effectiveness of Yinhuangsan (YHS) in patients with diabetic foot ulcers (DFU). Search Methods: Studies were selected from PubMed (1966 to January 2021), the Excerpta Medica Database (EMBASE) (1974 to January 2021), the Cochrane Library (1988 to January 2021), SinoMed (CBM) (1978 to January 2021), China Science and Technology Journal Data-base (VIP) (1994 to January 2021), Wanfang Data Knowl-edge Service Platform (1998 to January 2021) and the China National Knowledge Infrastructure (CNKI) (1984 to January 2021). No language restrictions were applied. Studies were identified and selected, and the data were extracted independently by two reviewers. The Cochrane Risk of Bias tool was used to assess the quality of studies. Revman 5.3 software was used for data synthesis and analysis. Results: Two studies were included based on the selection criteria. Two trials were of unsatisfied methodological quality and adopting different criteria to judge outcomes. As such, we were unable to perform a summary meta-analysis but concentrated on a narrative summary of results. Both the included studies had a low risk of incomplete data bias and selective reporting bias, while neither of them mentioned allocation concealment and blinding. Both two trials showed positive results favoring YHS compared with positive controls independently in total effective rate. One reported amputation rate, mortality and ulcer area change, and the other described ulcer healing rate. Neither of the trials stated adverse reactions and quality of life. Conclusions: There is currently inadequate evidence on evaluating YHS for DFU effectively, due to the paucity of randomized controlled trials and the low methodological quality in included studies. The safety of YHS remains unknown for lack of ample data on adverse events. Hence, YHS
\end{abstract}


should be applied with caution. More high quality randomized controlled trials (RCTs) expected to strengthen the evidence for the effectiveness and safety of YHS treating DFU are in demand. Standardized monitoring or a valid reporting system should be adopted to critically estimate adverse events and the safety in the future.

\section{Keywords}

Yinhuangsan, Diabetic Foot, Meta-Analysis

\section{Introduction}

Diabetic foot is one of the severe complications of diabetes mellitus (DM) and the common diseases of peripheral blood vessels, which is always combined with foot infection and lower extremity arterial occlusion. Severe cases may involve deep tissues [1].

Diabetic foot ulceration (DFU) is often persistent due to nerve abnormalities and vascular lesions in the lower extremities, local microcirculation as well as oxygen metabolism disorders [2]. A meta-analysis discovered that the prevalence rate of DFU all over the world is $6.3 \%$, while the prevalence rate varies greatly from country to country, ranging from $1.5 \%$ to $16.6 \%$ [3]. About $0.03 \%$ to $1.5 \%$ of patients with DFU suffered from amputation [4], and there is one patient with DFU who needs amputation every 20 seconds in the world currently [5] [6]. Studies showed that the mortality of DFU was $40 \%$ to $70 \%$ [7] [8].

In developed countries, DFU occupies $12 \%$ - 15\% health and medical resources for DM, while in developing countries, it occupies almost 40\% [9]. An American study indicated that the annual hospital expenses per DFU admission were $\$ 11,290$ [10]. So, DFU is bringing great pain to patients physically and mentally, as well as critical impact and burden to families and society.

At present, early prevention, professional diagnosis and timely treatment are of crucial importance in controlling DFU [11]. The treatments of DFU mainly include comprehensive medical therapy, local wound treatment, surgical operation as well as interventional therapy [12]. Comprehensive medical treatments consist of controlling blood glucose level, improving microcirculation, anti-inflammatory and so on [13]. Surgical treatments include vascular bypass grafting, negative pressure wound therapy with instillation, and stem cell transplantation to rebuild blood vessels [14]. However, the effectiveness of the above-mentioned treatments is not satisfying enough. A study showed that the recurrence rate of DFU patients over 50 years old was $31.6 \%$ within one year after treatment [15].

Given the above, more effective treatment options are expected to be imperatively taken into consideration. The use of traditional Chinese medicine (TCM) to treat DFU is becoming increasingly widespread these years, and evidence-based medicine has proven its effectiveness [16]. DFU is synonymous 
with gangrene and consumptive thirst in TCM, the main pathogenesis of which is as follows: deficiency of both Qi and Yin, blood blocking collaterals, and Exogenous damp-heat. As damp-heat and toxic-stasis are the major pathogenic factors of DFU, the concept of clearing heat and promoting diuresis is occupying a fairly prominent position in external therapy of TCM [17]. The traditional Chinese medicine Yinhuangsan (YHS) is composed of three traditional Chinese medicines: Cortex Phellodendri, earthworm and Resina Draconis, aiming at clearing heat and promoting diuresis, removing necrosis and promoting granulation, which may promote wound healing and reduce amputation rate to some extent [18].

In China, YHS has already been used in the treatment of DFU. The clear clinical effect of YHS has been reported in some reports. Li YS [19] described the total effective rate $(96.7 \%)$ in the treatment of DFU, concluding that YHS could obviously improve the clinical symptoms of DFU and promote the healing of DFU.

Although there are already some clinical studies on the efficacy of YHS, these studies are rare, and there is no systematic review to assess the efficacy and safety of YHS treating DFU currently. Hence, we evaluated the effectiveness of YHS in the treatment of DFU through systematic reviews and meta-analysis.

\section{Methods}

\subsection{Criteria for Considering Studies for This Review}

\subsubsection{Types of Studies}

All included studies were expected to be randomized controlled trials (RCTs) on the treatment of YHS for DFU regardless of language.

\subsubsection{Types of Participants}

Participants were male or female of any age or ethnic origin with DFU regardless of the severity of the disease and the involved sites. Participants were excluded if they had diabetic ketoacidosis and diabetic hyperosmosis syndrome, severe cardiopulmonary disease, allergy to multiple drugs, pregnant or breastfeeding.

\subsubsection{Types of Interventions}

All the trials that evaluated the effect of YHS on DFU were included. There was no limitation on the duration of treatment with YHS. The control group was given a placebo or blank or other treatment. The experimental group and the control group could receive the same dose and course of treatments.

\subsubsection{Types of Outcome Measures}

Primary outcome

- Total effective rate

- Amputation rate

- Mortality

- Ulcer healing rate

- Ulcer area change 
- The pain duration

- The pain intensity Secondary outcome

- Adverse events

- Quality of life

\subsection{Search Methods for Identification of Studies}

Comprehensive and exhaustive search strategies were formulated to identify all related studies regardless of language or publication status (published, unpublished, in press and in progress).

\section{Electronic Searches}

Complying with the guidelines from the Cochrane Collaboration, the following electronic databases were searched from their inception through January 2021:

- PubMed (1966 to January 2021)

- the Excerpta Medica Database (EMBASE) (1974 to January 2021)

- The Cochrane Library (1988 to January 2021)

- SinoMed (CBM) (1978 to January 2021)

- China Science and Technology Journal Database (VIP) (1994 to January 2021)

- Wanfang Data Knowledge Service Platform (1998 to January 2021)

- The China National Knowledge Infrastructure (CNKI) (1984 to January 2021)

The search terms used were as follows: "Yinhuangsan" and "diabetic foot ulcers" or "diabetic ulcer" or "tuoju" and "randomized controlled trial (RCT)".

The search strategy was adjusted for each database. In addition, the references of relevant articles and proceedings were examined for additional relevant literature. We did not limit publication language or type, including conference proceedings and theses, as long as they met our inclusion criteria. For example, the PubMed database retrieval strategy is shown as below.

(1) Diabetic Foot [MeSH] OR Foot, Diabetic [ti, ab.] OR Foot Ulcer, Diabetic [ti, ab.]

(2) Foot Ulcer [MeSH] OR Foot Ulcers [ti, ab.] OR Ulcer, Foot [ti, ab.] OR Ulcers, Foot [ti, ab.] OR Plantar Ulcer [ti, ab.] OR Plantar Ulcers [ti, ab.] OR Ulcer, Plantar [ti, ab.] OR Ulcers, Plantar [ti, ab.]

(3) diabet ${ }^{*}[\mathrm{ti}, \mathrm{ab}$.$] AND (ulcer { }^{*}$ [ti, ab.] OR foot [ti, ab.] OR wound $\left.{ }^{\star}[\mathrm{ti}, \mathrm{ab}].\right)$

((1) OR (2) OR (3)) AND((Yinhuangsan) OR (YHS))

\subsection{Data Collection and Analysis}

\subsubsection{Study Selection}

Two researchers independently screened the literature, evaluated the quality of the literature, extracted the data and cross-checked it. Different opinions were discussed and settled with the third party. Regarding study selection, the title was read first, and the abstract and the full text were read further to determine 
whether to include or not after the obviously irrelevant literature is excluded. If necessary, contact the original study author by email or telephone for undetermined but important information about the study.

\subsubsection{Assessment of Risk of Bias of Included Studies}

Risks of bias were assessed independently by two reviewers using the criteria described in the Cochrane Handbook for Systematic Reviews of Interventions 5.0.0 [20]. It is a two-part tool, addressing the six domains namely random sequence generation, allocation concealment, blinding, incomplete outcome data, selective outcome reporting and other issues. Disagreements were resolved either by consensus or by a third reviewer.

\section{1) Random sequence generation: assessment for selection bias}

The investigators describe a random component in the randomization process such as a random number table, a computer random number generator, coin tossing, or shuffling cards or envelopes and the like, which means their studies had a low risk of selection bias.

Trials that involved some systematic, non-random approach, such as odd or some rule based on hospital record number, or even by judgement of the clinicians, appeared to have a high risk of selection bias.

Trials in which the report did not provide sufficient information to permit judgement of "low risk" or "high risk" were referred to as an "unclear risk" of selection bias.

\section{2) Allocation concealment process: assessment of selection bias}

The allocation sequence was kept secure, for example by using central allocation, or sequentially numbered, sealed, opaque envelopes, so participants and investigators administering participants could not foresee assignment. These were referred to as a low risk of selection bias.

Studies in which the report mentioned 'concealed allocation' but was based on date of birth, case record number, an open random allocation schedule and any other explicitly unconcealed procedure, were recognized as having high risk of selection bias.

Trials in which the report did not provide sufficient information to permit judgement of "low risk" or "high risk" were regarded as a 'unclear risk' of selection bias.

3) Blinding of participants and personnel: assessment for performance bias

No blinding or incomplete blinding, but the outcome is unlikely to be influenced by lack of blinding, or double blinding in the report is unlikely could have been broken, which were considered as having a low risk of performance bias.

No blinding or incomplete blinding, and the outcome is likely to be influenced by lack of blinding, or double blinding in the report is likely could have been broken, which were recognized as having a high risk of performance bias.

Trials in which the report did not provide sufficient information to permit judgement of "low risk" or "high risk", or the study did not address this out- 
come, were regarded as an "unclear risk" of performance bias.

\section{4) Blinding of outcome assessment: assessment for detection bias}

No blinding of outcome assessment, but the outcome is unlikely to be influenced by lack of blinding, or blinding of outcome assessment in the report is unlikely could have been broken, which were considered as having a low risk of detection bias.

No blinding of outcome assessment, and the outcome is likely to be influenced by lack of blinding, or blinding of outcome assessment in the report is likely could have been broken, which were recognized as having a high risk of detection bias.

Trials in which the report did not provide sufficient information to permit judgement of "low risk" or "high risk", or the study did not address this outcome, were regarded as an "unclear risk" of detection bias.

\section{5) Incomplete data bias}

No missing outcome data, or the missing outcome data did not exert an unfavorable influence on true outcome, which were considered as having a low risk of bias.

The missing outcome data were likely to be related to true outcome, or potentially inappropriate of simple imputation, which were recognized as having a high risk of bias.

Trials in which the report provided insufficient reporting of exclusions to permit judgement of "low risk" or "high risk", or the study did not address this outcome, were referred to as an "unclear risk" of bias.

\section{6) Selective reporting bias}

Whether the study protocol is available, the expected outcomes reported in detail, were described as having a low risk of reporting bias.

If the study failed to report all primary outcomes, the trial was recognized as having high risk of reporting bias.

Trials in which the report did not provide sufficient information to permit judgement of "low risk" or "high risk" were regarded as an "unclear risk" of reporting bias.

\subsubsection{Data Extraction}

Each trial was assessed independently by two reviewers (Yichen Xiong, Haojie Huang). Information including trial design, types of participants, interventions and outcomes were extracted independently by two reviewers (Yichen Xiong, Haojie Huang). The data extraction details are as follows:

1) General information: published or unpublished, title, authors, reference or source, contact address, country, language, year of publication, setting.

2) Trial characteristics: design, duration of follow up, randomization process, allocation concealment, blinding (patients, clinicians, outcome assessors).

3) Intervention(s): intervention(s) (dose, route and follow-up time) and comparison intervention(s) (dose, route and follow-up time).

4) Patient characteristics: inclusion criteria, exclusion criteria, mean age, sam- 
ple size, diagnostic criteria, assessment of compliance, withdrawals and losses to follow up (reasons, description).

5) Outcomes: total effectiveness rate, complete healing rate, adverse events, amputation rate, changes in ulcer size, and ulcer healing time.

Discrepancies in the data extraction were resolved by referring back to the original article and consensus of all coauthors. When necessary, the reviewers contacted the primary authors.

All data in the included studies were binary outcomes, so the number of events and total number in each group were extracted or imputed. The formulation contents for the included studies were given in Table 1.

\subsubsection{Data Analysis}

Due to the clinical heterogeneity between included studies, we decided not to make a meta-analysis to calculate pooled effect size. Data were summarized in a narrative qualitative integration and different outcomes were analyzed separately.

If part of the data are available for meta-analysis, we will do the followings: The similar data were analyzed with Review Manager version 5.3 [21], which was used to process the data in the meta-analysis. Both dichotomous and continuous data are expected. Dichotomous data such as total effective rate and healing rate will be pooled using relative ratios (RR). Continuous data such as ulcer area change will be pooled using standardized mean difference (SMD). 95\% CI were calculated for data from included studies. Cochrane's $\chi^{2}$ test will be used to assess the degree of heterogeneity in included studies with significance set at $P<$ 0.1. Total variation across studies will be tested for using $P$ Statistic [22]. When the data did not reject the hypothesis of homogeneity $(P>0.1, P<50 \%)$, a fixed-effect model was used to calculate the summary OR, MD or SMD, and the 95\% CI. Otherwise, a random-effect model was utilized. Possible sources of heterogeneity will be assessed by sensitivity and subgroup analysis.

We have listed the reasons for exclusion of studies in Table 3 "Characteristics of excluded studies".

\section{Result}

\subsection{Description of Studies}

See: Table 2 "Characteristics of included studies"; Table 3 "Characteristics of excluded studies".

\subsubsection{Design of Included Studies}

See Table 2 "Characteristics of included studies". All included studies had a randomized controlled parallel study design. All the trials were conducted in China, one of which was published in 2001 and the other was reported in 2015.

\subsubsection{Patients of Included Studies}

In one study (Yang 2001), patients suffering from DFU were included according to the diagnostic criteria issued by WHO in 1985 , and DM duration must be 
Table 1. Contents of the formulations used in included studies.

\begin{tabular}{ccc}
\hline Study ID & Contents & Treatment \\
\hline Yang 2001 & YHS: Cortex Phellodendri, earthworm and Resina Draconis mixed at the ratio of 3:2:1. & $\begin{array}{c}\text { take some in sterile gauze for external } \\
\text { application, q.d for } 60 \text { days. } \\
\text { Li 2015 }\end{array}$ \\
YHS: Cortex Phellodendri 60g, earthworm 30g and Resina Draconis 10g. & extication q.d for 28 days. \\
\hline
\end{tabular}

Table 2. Characteristics of included studies.

Yang 2001

Methods

Participants

Interventions

Outcomes

Notes

Risk of bias

Bias

Random sequence generation

(selection bias)

Allocation concealment (selection Unclear risk bias)

Blinding (performance and detection Unclear risk bias)

Incomplete data bias

Selective reporting bias

Low risk

Other bias

Low risk

High risk

Randomized controlled parallel study design: the treatment group and the control group were 30 cases each according to the ratio of 1 to 1 .

Allocation concealment process and blinding were not mentioned.

Ethnicity: Chinese

Setting: inpatients

60 patients with DFU were all in line with the WHO diagnostic criteria for diabetes and complications in 1985 , and the course of DM must be more than 12 months.

Among them, 36 were male and 24 were female, 4 were insulin-dependent diabetes (IDDM), 58 were non-insulin-dependent diabetes (NIDDM), the average age was (64.21 \pm 8.1 ) years (41-80 years), and the average course was $(10.71 \pm 6.32)$ years ( $1-23$ years).

Exclusion criteria: not stated

Withdrawals and drop-outs: not stated

Characteristics of patients at baseline: similar.

Treatment group: Cortex Phellodendri, earthworm and Resina Draconis mixed at the ratio of 3:2:1. Bandaged with sterile gauze q.d for 30 days as a course of treatment ( 2 courses)

Control group: 1\% Ethacridine Lactate

1 Effective rate: 1 ) cure: the wound is completely covered with new derma; 2) remarkable improved: more than $1 / 2$ ulcer area closed up; 3 ) improved: $1 / 4$ - $1 / 2$ ulcer area closed up; 4 ) not improved: less than $1 / 4$ wound area closed up or enlarged

2 Clinical outcomes: not improved, amputated, dead

3 Ulcer area change: purulent exudate, red and swollen, pain, exfoliation of slough, the growth of granulation tissue

1 The formulation of YHS was provided by authors' department.

2 YHS was made by the author's hospital.

Reviewers' judgement

Low risk
Support for judgement

Adequate

Not mentioned

Not mentioned

No missing outcome data

All the expected outcomes reported in details

The formulation of YHS was provided by author's department, and it was made by the author's hospital. 
Methods

Participants

Intervention

Outcomes

Notes

Risk of bias

Bias

Random sequence generation

(selection bias)

Allocation concealment (selection

bias)

Blinding (performance and

detection bias)

Incomplete data bias

Selective reporting bias

Other bias

Randomized controlled parallel study design: randomly divided into two groups via a computer random number generator

Allocation concealment process and blinding were not mentioned.

Ethnicity: Chinese

Setting: inpatients

Western Medicine diagnostic criteria: 60 patients were in accordance with the report of Chinese Diabetes Society's (CDS) second meeting concerning diagnosis of DFU in 2000: DM patients whose acral skin appeared vesicle, blood blister, erosion, ulcer, gangrene or necrosis.

Chinese Medicine diagnostic criteria: 60 patients were in line with Guiding Principles for clinical research of new Chinese Medicine (1997), Surgery of traditional Chinese medicine (2007) and Diagnostic criteria of therapeutic effect of surgical diseases and syndromes in Chinese traditional medicine (ZY/TOO 1.1 - 94). Among them, 33 were male and 27 were female; mean age (58.34 \pm 10.72$)$ years ( $34-74)$; mean DM duration was (14.23 \pm 10.86 ) years (11 months - 26 years)

Exclusion criteria: not stated

Withdrawals and drop-outs: not stated

Characteristics of patients at baseline: similar.

Treatment group: YHS (Cortex Phellodendri 60 g, earthworm 30 g and Resina Draconis 10 g) q.d for 28 days Control group: Metronidazole and Glucose Injection q.d for 28 days

1 Therapeutic effect on DFU: reference to Nimodipine and therapeutic effect evaluation criterion on quantization integral

2 Healing rate: healing rate $=$ (ulcer area before treatment- not healed ulcer area)/ulcer area before treatment 3 Serum hs-CRP, TNF- $\alpha$, IL-1, VEGF, EGF, AGEs

4 Morphological observation of paraffin section of granulation tissue

1 The formulation of YHS was provided by authors' department.

2 YHS was made by the author's hospital.

3 The first author in this study was Yang BH's student.

Reviewers' judgement

Low risk

Unclear risk

Unclear risk

Low risk

Low risk

High risk

\section{Support for judgement}

Adequate

Not mentioned

Not mentioned

No missing outcome data

All the expected outcomes reported in details

The formulation of YHS was provided by author's department, and it was made by the author's hospital.

Table 3. Characteristics of excluded studies.

\begin{tabular}{cc}
\hline Study & Reason for exclusion \\
\hline Ji 2016 & The subjects were rats, not people. \\
Li 2016 & The subjects were rats, not people. \\
Li 2014 & Re Repetitive publication. \\
Yang 2003 & Repetitive publication.
\end{tabular}


over 12 months. The diagnostic criteria in the other study (Li 2015) were in accordance with the report of Chinese Diabetes Society's (CDS) second meeting concerning diagnosis of diabetic foot ulcers in 2000.

In one trial (Yang 2001), patients were aged from 41 to 80, and the duration of DM was 10 years on the average, while in the other trial (Li 2015), patients were aged from 34 to 74 , and the duration of DM was 14 years on the average.

\subsubsection{Interventions in the Included Studies}

In one study (Yang 2001), the authors used YHS as treated and used 1\% Ethacridine Lactate as control, while in the other study (Li 2015), they used YHS as treated and used Metronidazole and Glucose injection as control. The details about drug contents, routes and dosages are described in Table 1 "Contents of the formulations used in included studies" and Table 2 "Characteristics of included studies".

\subsubsection{Outcomes in the Included Studies}

No study assessed adverse events or quality of patients' life. The included two studies reported outcome measures in line with different criteria. See Table 3 "Characteristics of excluded studies".

\subsection{Literature Selection Process and Results}

Initially, we found 6 potentially relevant publications, and 4 publications [17] [23] [24] [25] were excluded after layer by layer screening. The reasons for the exclusion were shown in table-3. Finally, 2 RCTs [19] [26] were included, including 120 patients with diabetic foot ulcers, including 60 patients in the group of YHS treatment and 60 patients in the other treatment group. The retrieval process and results are shown in Figure 1.

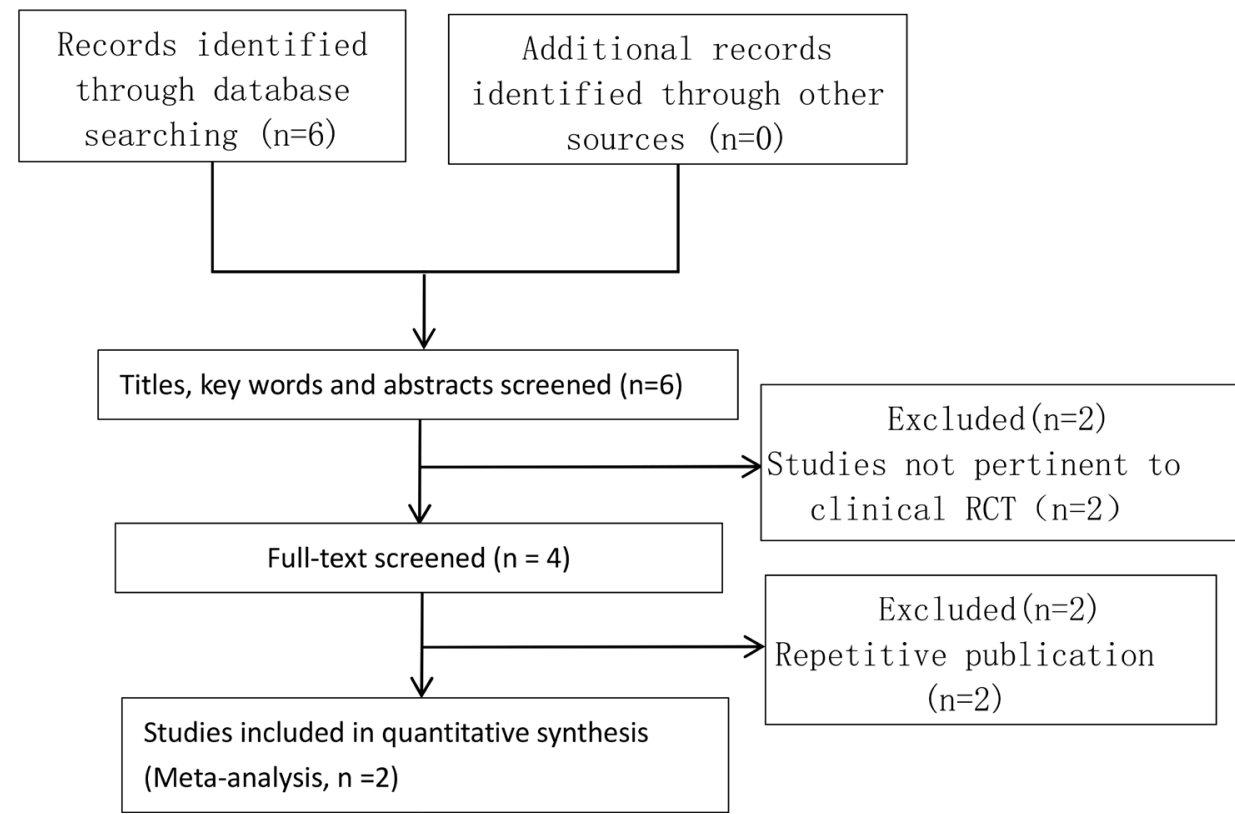

Figure 1. Literature selection process and results. 


\subsection{Risk of Bias in Included Studies}

\subsubsection{Randomization}

Both the included studies described randomization and had a low risk of selection bias. But neither of them mentioned allocation concealment, thus being considered as an "unclear risk" of selection bias.

\subsubsection{Blinding}

Neither of the included studies mentioned double blinding. Actually, it's always a challenge and obstacle for clinicians to mask participants, investigators as well as results assessors.

\subsubsection{Incomplete Data}

Neither of the included studies had withdrawals.

\subsubsection{Compliance Assessment}

Neither of the included studies reported on the methods to ensure compliance.

\subsubsection{Other Bias: Similarity of Comparison Groups at Baseline}

Both of the included studies had similar baseline between intervention group and comparison group, which was based on age, sex and DM duration and was comparable.

\subsection{Effects of Interventions}

Two included studies used the same intervention but adopted different diagnosis criteria as well as therapeutic evaluation. So we decided not to do a meta-analysis but concentrated on a narrative summary of results. There were no reported data on our selected primary outcomes of time for diabetic foot ulcers healing and adverse events, then we focused on the following outcome measures.

\subsubsection{Total Effective Rate}

This outcome measure was based on the four categories: cure, remarkable improved, improved and not improved. Yet two studies used different evaluation criteria, the detailed information about this criterion can be found in the "Description of studies" segment. Data on total effective rate of two studies are separately shown in Analysis 1.1. and Analysis 1.2.

1) Yang 2001

Yang's study showed positive results favoring YHS: YHS compared with $1 \%$ Ethacridine Lactate (RR 1.27; 95\% CI 0.82 to 1.97 ).

2) Li 2015

Li's study also showed convincing results supporting YHS: YHS compared with Metronidazole and Glucose Injection (RR 1.16; 95\% CI 0.98 to 1.38).

\subsubsection{Clinical Outcomes}

Clinical outcomes included amputation rate and mortality, and were reported only by one study (Yang 2001). See Analysis 2.1 for "amputation rate" and Analysis 2.2 for "mortality". Yang concluded the number of people who ampu- 
tated after two therapeutic courses (RR 0.29; 95\% CI 0.06 to 1.26) and reported there is no difference between Yihuangsan and 1\% Ethacridine Lactate regarding the number of dead patients (RR 1.00 ; $95 \%$ CI 0.15 to 6.64 ).

\subsubsection{Ulcer Change}

See Analysis 3.1 for "pain situation", Analysis 3.2 for "ulcer area change" and Analysis 3.3 for "ulcer healing rate". Yang described the number of people who suffered from ulcer pain (RR $0.29 ; 95 \%$ CI 0.10 to 0.83 ) and ulcer area change (SMD -0.54; 95\% CI -1.06 to -0.03 ). While Li reported the ulcer healing rate (SMD 1.03; 95\% CI 0.49 to 1.57 ).

Analysis 1.1. Comparison 1 Total effective rate, Outcome 1 Total effective rate in study (Yang 2001)

Review: YHS for DFU

Comparison: 1 Total effective rate

Outcome: 1 Total effective rate in study (Yang 2001)

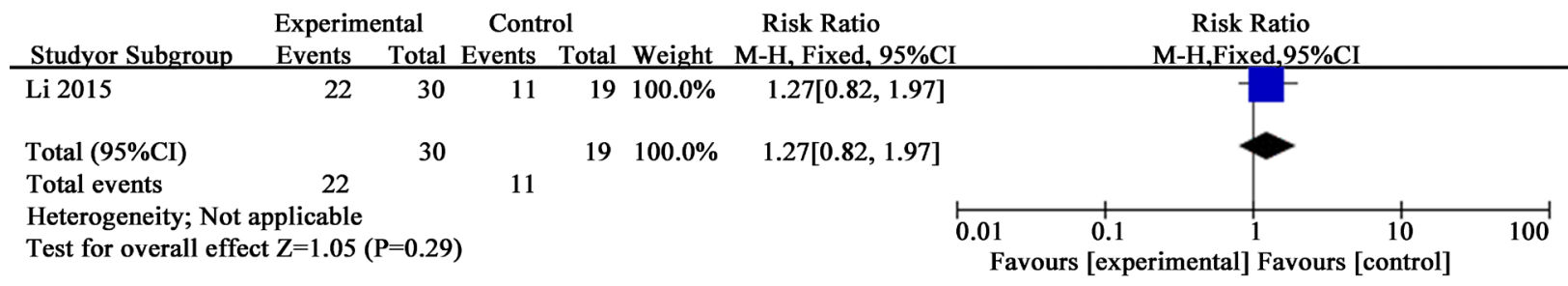

Analysis 1.2. Comparison 1 Total effective rate, Outcome 1 Total effective rate in study ( $\mathrm{Li} 2015)$

Review: YHS for DFU

Comparison: 1 Total effective rate

Outcome: 2 Total effective rate in study (Li 2015)

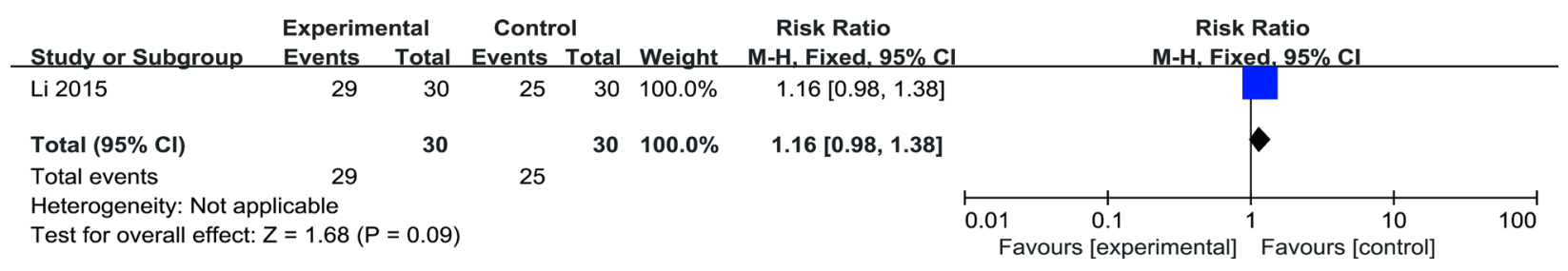

Analysis 2.1. Comparison 1 Clinical outcomes, Outcome 1 Amputation rate (Yang 2001)

Review: YHS for DFU

Comparison: 2 Clinical outcomes

Outcome: 1 Amputation rate (Yang 2001)

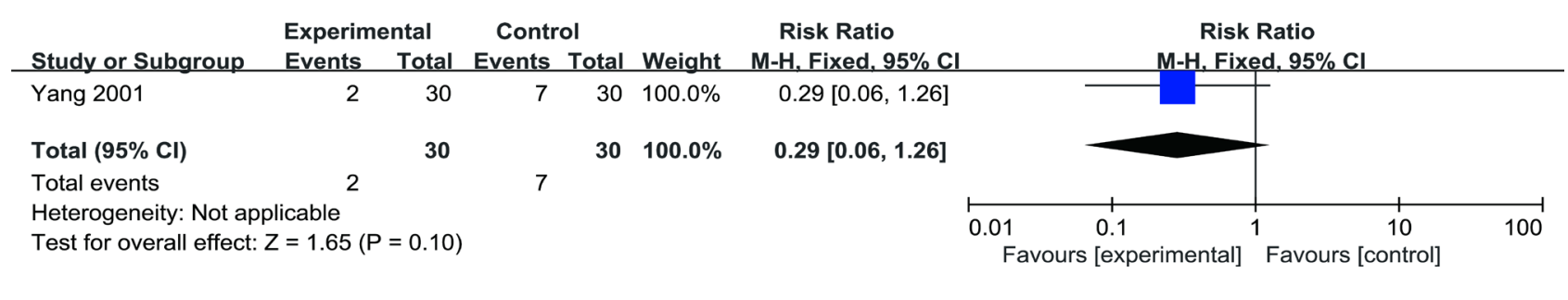


Analysis 2.2. Comparison 1 Clinical outcomes, Outcome 1 mortality (Yang 2001)

Review: YHS for DFU

Comparison: 2 Clinical outcomes

Outcome: 2 mortality (Yang 2001)

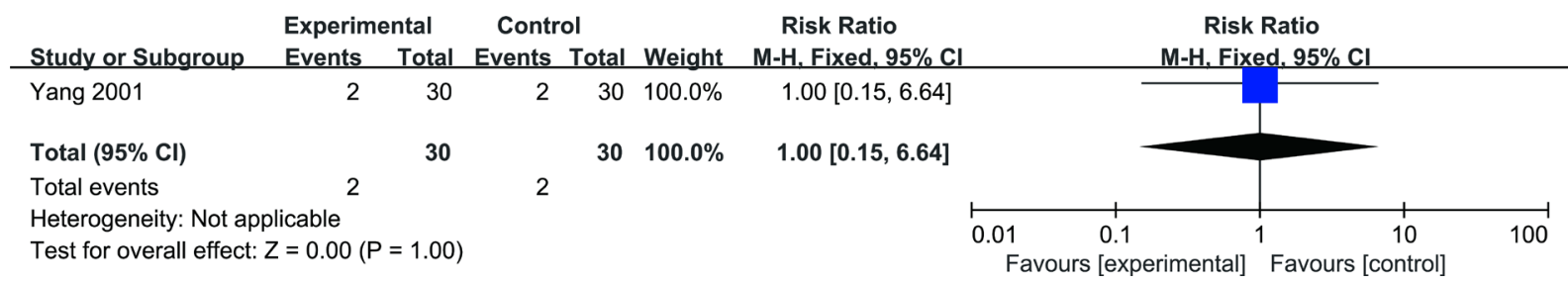

Analysis 3.1. Comparison 1 Ulcer change, Outcome 1 Pain situation (Yang 2001)

Review: YHS for DFU

Comparison: 3 Ulcer change

Outcome: 1 Pain situation (Yang 2001)

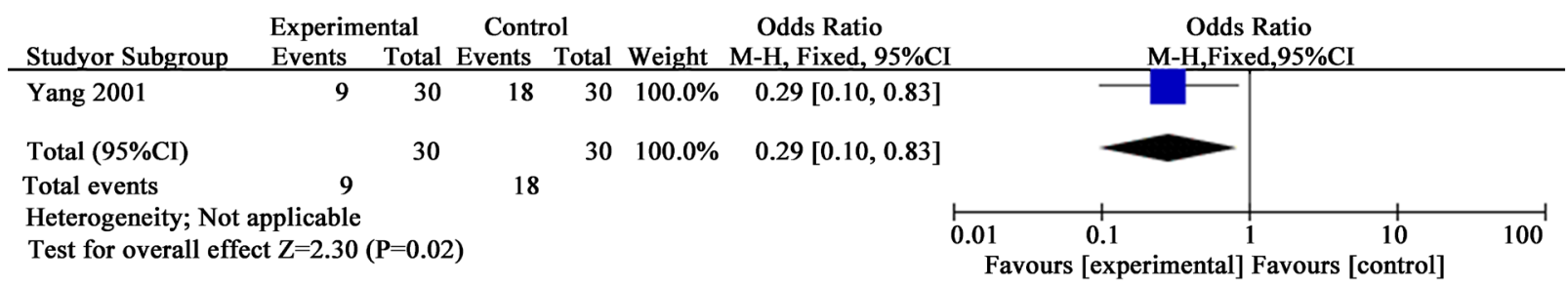

Analysis 3.2. Comparison 3 Ulcer change, Outcome 2 Ulcer area change (Yang 2001)

Review: YHS for DFU

Comparison: 3 Ulcer change

Outcome: 2 Ulcer area change (Yang 2001)

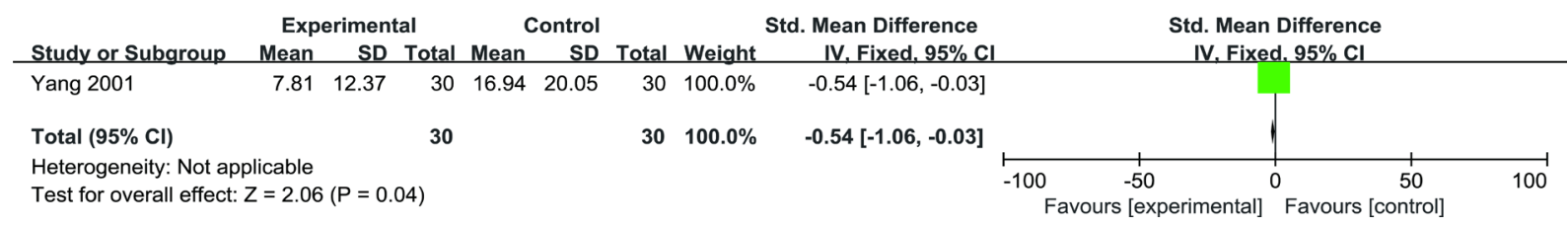

Analysis 3.3. Comparison 3 Ulcer change, Outcome 3 Ulcer healing rate (Li 2015)

Review: YHS for DFU

Comparison: 3 Ulcer change

Outcome: 3 Ulcer healing rate ( $\mathrm{Li} 2015$ )

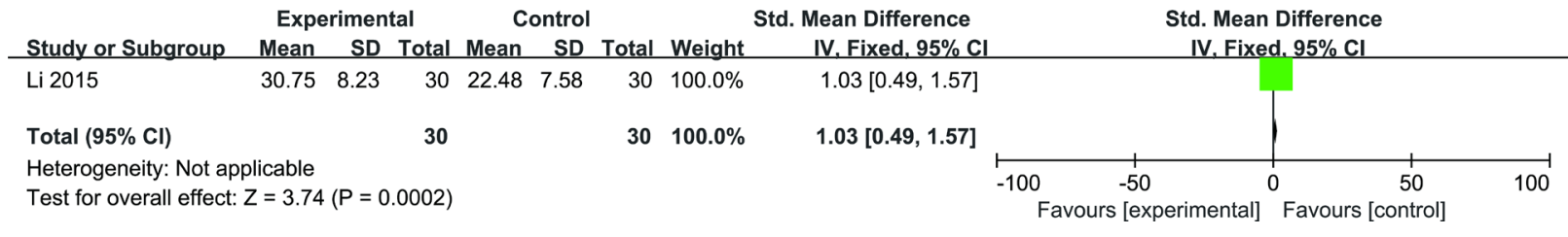




\section{Discussion}

\subsection{Summary of Main Results}

This systematic review examined the effectiveness of YHS for patients with DFU. Two studies reported total effective rate, but no study provided us with data on adverse events such as allergy or measured patient quality of life. One study was able to provide positive results for ulcer healing rate. The other study was able to demonstrate limited benefit of YHS compared with $1 \%$ Ethacridine Lactate in terms of amputation rate and mortality. We were unable to determine if YHS was more effective than the positive controls, mainly because the trials were small with wide confidence intervals and insufficient statistical power. Overall, studies of YHS for DFU lack enough power to provide credible estimates of the effects.

\subsection{Quality of the Evidence}

The evidence from the included studies was of low quality.

1) Publication bias may exist because only Chinese language publications were found and just two were included.

2) Among the 6 papers that we retrieved for further details, two studies were not pertinent to clinical RCTs, and other two studies were repetitive publication. We identified only two of the retrieved studies as true RCTs and meet our inclusion criteria. Both described randomization but neither of them mentioned allocation concealment and blinding. Only one of them had a source of funds. No analysis was reported based on intention to treat. The above questions could result in bias and exaggeration of the efficacy of the treatment group.

3) Neither of the two studies used a placebo as control. One study used 1\% Ethacridine Lactate and the other used Metronidazole and Glucose injection as positive controls, which may result in false positive findings for YHS was considered effective for DFU, particularly if clinicians know that a 'positive' drug was used in the trial and the purpose of the study was to demonstrate the same effect as the control.

4) We found that YHS was prepared by the Yang and Li themselves in their hospital. Also, the authors acted as the main players, including formulation designer, trial designer, implementer and assessors, which probably lead to a high possibility of a bias.

Moreover, YHS as a treatment for DFU has not been widely accepted in China, and pharmacological effect of YHS cannot be clearly specified. This is in significant contrast to Western medicine, in which the chemical constituents, quantities and the percentages of any impurities or contaminants are precisely known; and the variation between different production batches is kept within specified limits. This variation is a factor that may contribute to heterogeneity between two study results.

5) Neither of the studies conformed to the criteria laid down in the CONSORT statement [27]. The results for the interventions did not come close 
to reaching a convincing conclusion as to the effectiveness of YHS compared to another certain Chinese patent medicine or a herbal preparation. If more high quality studies on the same interventions had been found, the effectiveness of YHS could have been evaluated more reliably using meta-analysis.

6) Some of the outcome definitions adopted by this review, and employed by the included studies, were based on a subjective judgement by individual clinicians, thus making the results somewhat unreliable. And specific adverse effects were not adequately reported by any of the studies.

7) It is difficult to compare the outcomes of YHS with standard 'Western' clinical outcomes, for the outcomes were relying on the subjective interpretation of the clinicians in their observations or subjective feelings of patients. For example, the included studies regarded purulent exudate, red and swollen, pain, exfoliation of slough, the growth of granulation tissue as the criteria on outcomes of ulcer area change.

\section{Conclusions}

TCM categorizes DFU into "Xiao Ke" and "Tuo Ju", a condition owing to poor nourishment of extremities. It's universally considered the basic pathogenesis of diabetic foot ulcers to be a process that involves obstruction of blood vessels and superficial attacks of damp-heat, thus the therapeutic principles covering mobilizing stagnant blood, dissolving impediments and obliterating damp-heat in Chinese medicine.

YHS is such a prescription that consists of three traditional Chinese medicines, namely Cortex Phellodendri, earthworm and Resina Draconis, which may promote wound healing and reduce amputation rate to some extent [28]. In the records of TCM, YHS has a definite curative effect on healing DFU.

There are some limitations to the present analysis. First and foremost, neither of the included trials specified how they handled concealment and blinding, which is bound to give rise to bias and exaggeration of the efficacy of YHS. For most clinicians in China, random sequence generation and allocation concealment process are expected to be emphasized and expounded expressly. We are in need of well-designed, randomized and multi-center clinical trials in the days to come. Also, the specific appearance and property of YHS may make blinding difficult. Drug types should be consistent in both of the comparison groups. Secondly, the selected trials were sporadic and small-sampled. More studies with large numbers of participants and good reporting to provide more compelling evidence are in demand.

In a nutshell, there is currently inadequate evidence on evaluating YHS for DFU effectively, on account of the paucity of randomized controlled trials conducted and the low methodological quality in these studies. Given the above-mentioned respects, YHS should be applied with caution. Besides, the safety of YHS remains unknown for lack of ample date on adverse events.

Also, clinical trials should come to endpoints of amputation coupled with 
death and, from the patients' perspective, quality of life. Both require long-term follow up. Standardized monitoring or a valid reporting system should be adopted to critically estimate adverse events and the safety.

Nevertheless, we believe that this study reflects the basic trends of the pharmaceutical efficacy of YHS for DFU, and some guidance on future normative trials. The multidisciplinary therapeutic approach, which concerns a combination of TCM and Western medicine, surgical management, education regarding DFU care, and psychological counseling, is needed by patients with DFU.

\section{Ethical Approval}

This study was based on previously published studies, so ethical approval and patient consent are not relevant.

\section{Authors' Contributions}

Yichen Xiong, Haojie Huang, Yaochen Li, Guohua Jiang and Lin Du had full access to all the study data and take responsibility for its integrity and the accuracy of the analysis. Yichen Xiong and Haojie Huang were responsible for the study concept and design, data acquisition and extraction. The assessment of bias risk was performed by Yichen Xiong and Haojie Huang; data analysis and interpretation were performed by Yichen Xiong. Yichen Xiong, Haojie Huang, Yaochen Li, Guohua Jiang and Lin Du contributed to the revision of the manuscript. All authors have read and approved the final manuscript.

\section{Acknowledgements}

This project was supported by national undergraduate training program for innovation and entrepreneurship of China (no. S201910572128). Protocol registered ID: CRD42020169597.

\section{Conflicts of Interest}

The authors declare no conflicts of interest regarding the publication of this paper.

\section{References}

[1] Cuttica, D.J. and Philbin, T.M. (2010) Surgery for Diabetic Foot Infections. Foot and Ankle Clinics, 15, 465-476. https://doi.org/10.1016/j.fcl.2010.03.006

[2] Basu, M.S., Jayashree, B.S. and Shenoy, R.R. (2018) Epigenetic Modulation of Macrophage Polarization-Perspectives in Diabetic Wounds. Journal of Diabetes and Its Complications, 32, 524-530. https://doi.org/10.1016/j.jdiacomp.2018.01.015

[3] Zhang, P., Jing, Y., et al. (2017) Global Epidemiology of Diabetic Ulceration: A Systematic Review and Meta-Analysis. Annals of Medicine, 49, 106-116. https://doi.org/10.1080/07853890.2016.1231932

[4] Lazzarini, P.A., Hurn, S.E., Fernando, M.E., et al. (2015) Prevalence of Foot Disease and Risk Factors in General Inpatient Populations: A Systematic Review and Meta-Analysis. BMJ Open, 5, e008544. https://doi.org/10.1136/bmjopen-2015-008544 
[5] International Diabetes Federation (2017) IDF Diabetes Atlas. 8th Edition, Brussels.

[6] Bakker, K., Apelqvist, J., Lipsky, B.A., et al. (2016) The 2015 IWGDF Guidance Documents on Prevention and Management of Foot Problems in Diabetes: Development of an Evidence-Based Global Consensus. Diabetes/Metabolism Research and Reviews, 32, 2-6. https://doi.org/10.1002/dmrr.2694

[7] Jupiter, D.C., Thorud, J.C., Buckley, C.J. and Shibuya, N. (2016) The Impact of Foot Ulceration and Amputation on Mortality in Diabetic Patients. I: From Ulceration to Death, a Systematic Review. International Wound Journal, 13, 892-903. https://doi.org/10.1111/iwj.12404

[8] Brennan, M.B., Hess, T.M., Bartle, B., Cooper, J.M., Kang, J., Huang, E.S., et al. (2017) Diabetic Foot Ulcer Severity Predicts Mortality among Veterans with Type 2 Diabetes. Journal of Diabetic Complications, 31, 556-561.

https://doi.org/10.1016/j.jdiacomp.2016.11.020

[9] Raghav, A., Khan, A., Labala, R.K., et al. (2018) Financial Burden of Diabetic Foot Ulcers to World: A Progressive Topic to Discuss Always. Therapeutic Advances in Endocrinology and Metabolism, 9, 29-31.

https://doi.org/10.1177/2042018817744513

[10] Hicks, C.W., Selvarajah, S., Mathioudakis, N., et al. (2016) Burden of Infected DFU on Hospital Admissions and Costs. Annals of Vascular Surgery, 33, 149-158. https://doi.org/10.1016/j.avsg.2015.11.025

[11] Diabetes Branch of Chinese Medical Association, Infectious Diseases Branch of Chinese Medical Association, Tissue Repair and Regeneration Branch of Chinese Medical Association (2019) Guidelines for the Prevention and Treatment of Diabetic Foot in China (2019 Edition). Chinese Journal of Diabetes Mellitus, 11, 92-108.

[12] Fan, G.J., Zhao, L., Tang, X.Y., Li, Z., Shen, Y.D., Yuan, Q., Pang, G.M. and Gao, H.L. (2011) Diagnosis and Treatment Standard of Traditional Chinese Medicine for Diabetic Foot. World Journal of Integrated Traditional and Western Medicine, 6, 618-625.

[13] Hu, C.H. (2017) Clinical Study of the Clinical Characteristics and Comprehensive Medical Treatment of Diabetic Foot. World Latest Medicine Information, 17, $57+59$.

[14] You, H., Yang, Q.Z., Liu, P.K. and Zhu, Y.L. (2019) Progress in Surgical Treatment of Diabetic Foot Diabetes. New World, 22, 193-196.

[15] Cheng, Y.X., Zhao, F.H., Zhu, P., et al. (2019) Risk Factors of Recurrent Diabetic Foot Ulcers. Beijing Medical Journal, 41, 1024-1027.

[16] Tan, L.Z., Shi, Q.Y., Liu, C.X., Zhang, J.H., Wang, H. and Zhai, J.B. (2018) Traditional Chinese Medicine Injections in the Treatment of Diabetic Foot: A Systematic Review and Meta-Analysis. Evidence-Based Complementary and Alternative Medicine, 2018, Article ID: 4730896. https://doi.org/10.1155/2018/4730896

[17] Li, Y.S., Yang, B.H. and Ji, L.Y. (2016) Effect of Yinhuangsan on Morphology of Ulcer Wound and Ulcer Healing Factors on Diabetic Rats. Global Traditional Chinese Medicine, 9, 10-14.

[18] Yu, M., Zhang, J., Zhao, J., et al. (2017) Study of the Effect of Heparan Sulfate on Wound Healing in Diabetic Rats. Chinese Journal of Vascular Surgery (Electronic Version), 2, 138-142.

[19] Li, Y.S. and Yang, B.H. (2015) Study on the Correlation between AGEs and Healing Factors in the Treatment of Diabetic Foot Ulcer. World Science and Technology-Modernization of Chinese Medicine, 17, 350-355. 
[20] Higgins, J.P.T. and Green, S. (2008) Application of Quality Assessment Criteria. In: Higgins, J.P.T. and Green, S., Eds., Cochrane Handbook for Systematic Reviews of Interventions, John Wiley \& Sons, Chichester, 174-191. https://doi.org/10.1002/9780470712184

[21] The Cochrane Collaboration (2012) Review Manager (RevMan) Computer Program. Version 5.2. The Nordic Cochrane Centre, Copenhagen.

[22] Higgins, J.P., Thompson, S.G., Deeks, J.J. and Altman, D.G. (2003) Measuring Inconsistency in Meta-Analyses. BMJ, 327, 557-560. https://doi.org/10.1136/bmj.327.7414.557

[23] Ji, LY. (2016) Effect of Traditional Chinese Medicine "Yinhuangsan" on the Relevant Factors of Diabetic Ulcer Rats. Peking University, Beijing.

[24] Li, Y.S. (2014) Study on the Correlation between AGEs, Inflammatory Factors and Growth Factors in the Healing Process of Diabetic Foot Ulcer with "Yinhuangsan". Peking University, Beijing.

[25] Yang, B. (2003) Clinical Study on Treatment of Diabetic Foot Ulcer with Yinhuang Coating Agent. 11 th National Academic Exchange Meeting of the Selection Branch of Chinese Society of Integrated Traditional and Western Medicine, 23-24.

[26] Yang, B.H., Zhang, Y.S. and Zhao, S.S. (2001) Clinical Observation on the External Treatment of Diabetic Foot Ulcer with Yinhuangsan. Journal of External Therapy of Traditional Chinese Medicine, No. 4, 22-23.

[27] Moher, D., Schulz, K.F. and Altman, D.G. (2001) The CONSORT Statement: Revised Recommendations for Improving the Quality of Reports of Parallel-Group Randomised Trials. The Lancet, 357, 1191-1194. https://doi.org/10.1016/S0140-6736(00)04337-3

[28] Lipsky, B.A., Berendt, A.R., Deery, H.G., et al. (2006) Diagnosis and Treatment of Diabetic Foot Infections. Plastic and Reconstructive Surgery, 117, 212S-238S. https://doi.org/10.1097/01.prs.0000222737.09322.77 